九州大学学術情報リポジトリ

Kyushu University Institutional Repository

\title{
System optimization without numerical target
}

\section{高木，英行}

Department of Acoustic Design, Kyushu Institute of Design

http://hdl. handle. net/2324/4486259

出版情報: Proceedings of North American Fuzzy Information Processing, pp.351-354, 1996-06-19. IEEE バージョン :

権利関係 : 


\title{
System Optimization Without Numerical Target
}

\author{
Hideyuki TAKAGI \\ Dept. of Acoustic Design, Kyushu Institute of Design \\ 4-9-1, Shiobaru, Minami-ku, Fukuoka 815 Japan \\ TEL +81-92-553-4555, FAX +81-92-553-4569, takagi@kyushu-id.ac.jp
}

\begin{abstract}
This paper describes the importance of next challenging task, system optimization based on human preference or subjective evaluation, and shows how to challenge the task. As one of this type of approaches, interactive GA is introduced, and its advantages and disadvantages are discussed. To solve the disadvantages, this paper proposes two methods to reduce the burden of interactive GA operators, and evaluates their performances. Subjective tests have shown that the proposed methods are significantly better for human operators than conventional interface from statistical test point of view.
\end{abstract}

\section{INTRODUCTION}

Most cases of auto-designing or auto-adjusting systems have numerical specifications. Using the numerical targets, error between system outputs and the targets is calculated, and the systems are adjusted to minimize the error (see Figure 1 (a)). Control systems and adaptive systems are some of them.

Figure 2 is a sample that is popular in cooperative systems in soft computing and that is widely used in consumer products, such as washing machines, rice cookers, vacuum cleaners, photo copiers, and so on [12]. Since the fuzzy system in Figure 2 has numerical specification as supervised data, the neural network in the figure can adjust parameters of the fuzzy system to minimize the error between the actual output and specification of the fuzzy system.

However, some systems whose outputs are evaluated by only human do not have their numerical targets (see Figure 1 (b)). Suppose to design a vehicle controller which lets passengers feel comfortable, to design an air conditioning controller which lets people in a room feel comfortable, to adjust the parameters of a music synthesizer to match its sound to the sound image in your mind, or to create computer graphics which matches to the given design concept. Only human can evaluate the output of these systems based on his/her psychological measure, subjective sense or preference.

Although human can evaluate the outputs of these systems, it is hard for the human to adjust parameters of the systems to optimize their outputs. To automate the designing systems, we need to combine optimization techniques for optimizing the parameters and human evaluation for optimizing navigation. Unlikely Figure 2, neural networks cannot be used in this case, because we cannot get gradient information from human evaluation space.

One of solution is to use genetic algorithms (GAs) with human subjective evaluation, because the GA does not request many information of searching spaces, such as gradient information. This optimization approach based on human evaluation is interactive GA.

Interactive GA has applied to several tasks. However, there are many problems to use it practically. This paper describes current issues that we must solve and proposes some solutions.

\section{Interactive GA}

Interactive GA is a genetic algorithm whose fitness function is replaced by human evaluation. Figure 3 shows the diagram of the interactive GA that is applied to optimize a synthesizer, a CAD, or CG. GA decides parameters of these target systems. Each system synthesizes sound, figure, or image based on the parameters. Human operator evaluates how the output is close to given concept or his/her preference and returns a numerical evaluation value on his/her psychological measure to the GA. Note that the numerical values that are feed-backed to GA is semantically different from the numerical target that is discussed in the previous section.

The advantage of the interactive GA is that this technology gives one solution to tasks of auto-tuning or optimizing systems whose performances can be evaluated by only human. There had been no technology which automates the optimization of any systems 


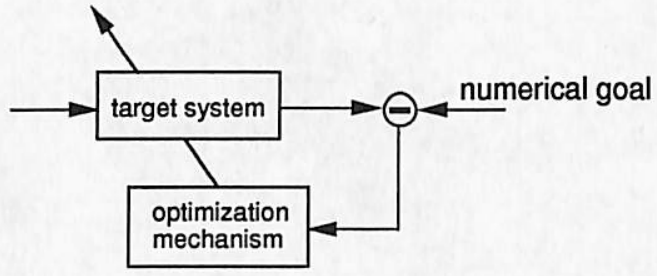

(a)

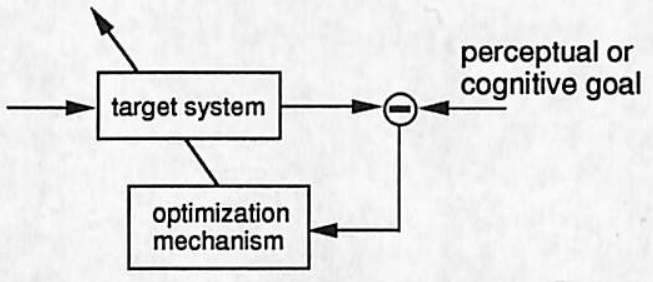

(b)

Figure 1: Auto-system optimization: (a) optimization based on numerical goal, and (b) optimization based on human evaluation

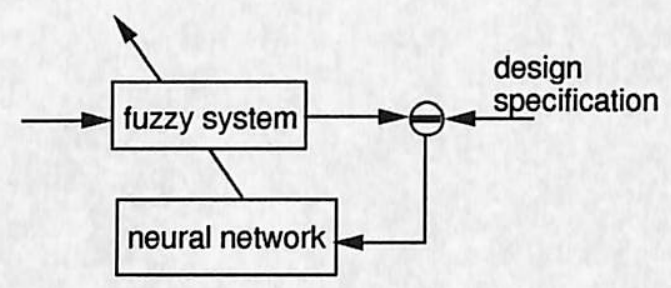

Figure 2: Optimizing fuzzy system using a neural network

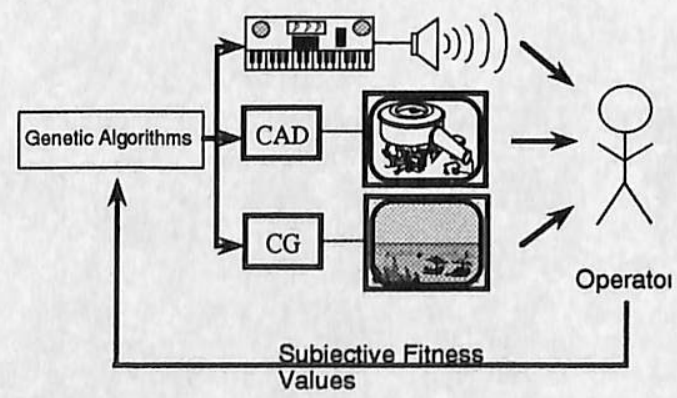

Figure 3: System flow of interactive GA whose performances are evaluated by the perceptual or cognitive measure such as beauty, comfort, conceptually well matched, or preference except manual tuning. The interactive GA expands the auto-tuning area from systems which have numerical goal to those which have perceptual or cognitive goal.

The disadvantage of the interactive GA is that physical and psychological burden of human operator is rather big. The interactive GA requires human operators to cooperate with a tireless computer. Since human operators are requested to evaluate and input an evaluation score to each individual in each generation. It is very tired and takes long time for human to score many individuals in many generations based on his/her psychological measure, while it is an easy job for a computer to run GA process for 1,000 or 10,000 generations

As there are tasks which cannot be solved without the interactive GA, what we must do is to develop techniques to reduce the human burden that the interactive GA requires. This is one of main topics of this paper and is discussed in section 4 .

Interactive GA has been mainly applied to design of images and figures. Some of them are: montage face image generation [3], designing shapes of bug biomorphs [11], creating images, evolving expressions that specify particular sequences of image-processing functions [10], combining interactive evolution with constructive solid geometry techniques to create computer renderings of 3-D forms ("virtual sculptures") [15], general-purpose interactive graphic layout system based on GA [7], the design of a double curvature concrete arch dam [8], line drawing and application to face drawing [1], and the decision supporting system for aesthetic design of cable-stayed bridges [6].

There are a few applications of the interactive GA to time sequential tasks, such as Jam session [2], generating rhythms of percussion instruments [4], and speech processing [17].

\section{Interactive Ga for Tasks Which HaVe Sequential Output}

It is physically and psychologically hard for interactive GA operators to use the interactive GA for tasks whose output is shown sequentially, because they have to compare current output with past one in their memory. This is why there are few applications of the interactive GA to time sequential tasks.

Our question is whether the interactive GA is effective for the tasks of time sequential presentation. It is not difficult to change the presentation procedure of the system output from spatial to time sequential. But, applicability and effectiveness are different issues.

To answer the question, we applied the interactive 
Table 1: Statistical test with the Sheffe's method of paired comparisons. $D$ is the original distorted speech; $X_{k}$ is the speech which is recovered by a recovering filter made by the operator $\mathrm{X}$ with $k$-th generation of interactive GA. $\bigcirc$ means that the difference between 2 average preferences of 32 subjects is significant $(p<$ $0.01)$.

\begin{tabular}{|c|c|c|c|}
\hline \multirow{2}{*}{ paired comparison } & \multicolumn{3}{|c|}{ operators } \\
\cline { 2 - 4 } & $\mathrm{A}$ & $\mathrm{B}$ & $\mathrm{C}$ \\
\hline$D$ vs. $X_{10}$ & $\bigcirc$ & $\bigcirc$ & $\bigcirc$ \\
\hline$D$ vs. $X_{20}$ & $\bigcirc$ & $\bigcirc$ & $\bigcirc$ \\
\hline$D$ vs. $X_{40}$ & $\bigcirc$ & $\bigcirc$ & $\bigcirc$ \\
\hline
\end{tabular}

GA to speech processing and statistically tested its effectiveness [17]. The main objective of this research was not apply the interactive GA to new applications task, auto-designing a filter with subjective hearing, but to quantitatively evaluate the effectiveness of the interactive GA whose system outputs are time-sequentially presented.

The task is to make a filter that recovers distorted speech based on human hearing. The interactive GA decides the coefficients of the FIR filter. After the designing, the original distorted speech and recovered speech are evaluated by subjects without the GA operator. If the interactive GA has no effect for this task, there would not be significant difference between two speeches even if the GA operator feels the recovered speech was better quality for him or her.

The subjective test has been conducted with 32 subjects. They compare the original distorted speech with nine speeches that are recovered with three FIR filters generated in 10th, 20th, and 40th generations of three GA operators. Table 1 shows the statistical test of the Sheffé's method of paired comparison. Filters of any three generations of three GA operators can recover distorted speech significantly better than original distorted speech.

From this result, it can be said that there is, at least, a task whose system outputs are time-sequentially presented and that the interactive GA can effectively work. Once we can say the effectiveness of the interactive GA to time-sequential tasks, making the interactive GA practical is the next task. The section 4 discusses this issue.

\section{Improving Interface for Practical INTERACTIVE GA}

To solve the problem of human burden mentioned in the previous section, it is important to improve input and output (or presenting) interfaces of the interactive GA. Speed-up of GA searching is useful to reduce the burden of the interactive GA operator. But improving interface is especially important to sold the problem that is caused by the time sequential presentation.

We propose two methods to improve the input interface by using discrete fitness values. The discrete here means psychological sense. Suppose to rate the given outputs of a system that we want to optimize with 100 levels and 5 levels. Although 100 levels are discrete mathematically, we can say it is continuous on psychological measure. On the other hand, we can usually distinguish the difference of given figures or sounds in five grades; rating with five levels can be said as input method of discrete fitness values.

The advantage of this method is that GA operators need not care about small difference and gives same fitness values; imagine how it requests human burden to distinguish the difference between sounds of 67 points and 68 points.

The disadvantage might be to result slow GA search convergence due to quantization error in fitness values. Our simulation results that it is true if the GA search reaches to hundreds-th generation order. Fortunately, (and unfortunately) we cannot use the interactive GA for long generation search because of human tiredness. From the practical point of view, the simulation has shown that the quantized noise caused by the proposed method does not give bad influence to GA convergence in several or several dozens generations [13].

To evaluate how the proposed input method using discrete fitness values is easy to use or reduce human burden, we compare it with the conventional input method through subjective tests. We use two proposed methods: input method of discrete fitness values only and combinational input method of both discrete and continuous fitness values. We also combine the time sequential presentation that shows only one output each time by each and spatial presentation that shows all outputs at once.

The task is to make a drawing face that matches face $e$ in our mind. The number of subjects is 18 . Two subjective tests are used: the method of successive categories [5] and the Ura's variation of Sheffe's method of paired comparisons $[9,16]$.

Table 2 shows the result of statistical test that is obtained from analysis of variance for the Sheffé's method of paired comparisons. We can conclude that the proposed method is significantly better for interactive GA than the conventional input method that has continuous fitness values. It also concludes that allowing interactive GA operators to choose either the proposed method and the conventional method is better. 
Table 2: Statistical test for significance psychological differences obtained by the Sheffé's method of paired comparisons. P1 and P1 are fitness value input methods. P1 is discrete one (proposed method \#1); P2 is combination of discrete/continuous ones (proposed method \#2) with sequential presentation; $A$ is continuous one with sequential presentation (conventional method); B is continuous one with simultaneous presentation (conventional method).

\begin{tabular}{|c||c|c||c|}
\hline & A vs. P1 & P1 vs. B & P1 vs. P2 \\
\hline test & significant & not significant & significant \\
result & $(p<0.01)$ & $(p<0.05)$ & $(p<0.01)$ \\
\hline
\end{tabular}

\section{Conclusion and Future Works}

As the next target of system optimization, system optimization based on human preference or subjective evaluation, which does not have numerical goal, becomes important. Interactive GA is one of tools to challenge this target. This paper has proposed methods to make the interactive GA practical by improving its input interface and shown how they significantly improve the interactive GA interface through subjective tests and statistical analysis. This is one of steps to make practical interactive GA for the challenging task, system optimization without numerical goal.

This paper stresses that improving interface is important to use the interactive GA practically and proposes new input methods. We have started to evaluate new method to improve presentation interface. If a system can learn evaluation characteristics of the interactive GA operators, it must help human evaluation and reduce human burden. We have introduced an online type of a neural network. From the simulation, we have found that the evaluation output of the neural networks is surely better than random order of GA outputs [14]. The next step is to evaluate this method quantitatively through subjective test as shown in this paper.

\section{REFERENCES}

[1] Baker, E. and Seltzer, M., "Evolving line drawings," Graphics Interface'94 Proc., Banff, Alberta, Canada, edited by Wayne A. Davis and Barry Joe. Morgan Kaufmann Publishers, pp.91-100 (May, 1994).

[2] Biles, J. A., "GenJam: a genetic algorithm for generating jazz solos," Int'l Computer Music Conference (ICMC'94), pp.131-137, Aarhus, Denmark (1994)

[3] Caldwell, C. and Johnston, V. S., "Tracking a criminal suspect through "face-space" with a genetic algorithm," 4th Int'l Conf. on Genetic Algorithms (ICGA'91), San Diego, CA, USA, pp.416-421 (July, 1991)
[4] Damon. H.: "Generating rhythms with genetic algorithms," Int'l Computer Music Conference (ICMC'94), Aarhus, Denmark, (1994)

[5] Dixon, W. J. and Massey, F. J., "Introduction to statistical analysis," McGraw-Hill, New York, pp.342-343 (1951).

[6] Furuta, H., Maeda, K., and Watanabe, E, "Application of genetic algorithm to aesthetic design of bridge structures," Microcomputers in Civil Engineering, Vol.10, No.6, pp.415-421 (1995)

[7] Masui, T., "Graphic object layout with interactive genetic algorithms," 1992 IEEE Workshop on Visual Languages, Los Alamitos, CA, USA, pp.74-80 (1992).

[8] Parmee, I. C., "The concrete arch dam: an evolutionary model of the design process," Int'l Conf. on Artificial Neural Nets and Genetic Algorithms, Innsbruck, Austria, pp.14-16 (April, 1993).

[9] Sheffé, H., "An analysis of variance for paired comparisons," Am. Statis. Asoc. J., Vol.47, pp.381- (1952).

[10] Sims, K., "Artificial evolution for computer graphics," Computer Graphics, Vol.25, No.4, pp.319-328 (July, 1991).

[11] Smith, J. R., "Designing biomorphs with an interactive genetic algorithm," 4th Int'l Conf. on Genetic Algorithms (ICGA'91), San Diego, CA, USA, pp.535-538 (July, 1991)

[12] Takagi, H., "Applications of Neural Networks and Fuzzy Logic to Consumer Products," ed. by J. Yen, R. Langari, and L. Zadeh, in Industrial Applications of Fuzzy Control and Intelligent Systems, Ch.5, pp.93106, IEEE Press, Piscataway, NJ, USA (1995)

[13] Takagi, H. and Ohya, K., "Discrete Fitness Values to Improve Human Interface of Interactive GA," IEEE 3rd Int'l Conf. on Evolutionary Computation (ICEC'96), Nagoya, Aichi, Japan (May, 1996)

[14] Hideyuki Takagi, "Interactive GA for System Optimization: Problems and Solution," 4th European Congress on Intelligent Techniques and Soft Computing (EUFIT'96), Aachen, Germany (Sept.2-5, 1996) (will appear)

[15] Todd, S. and Latham, W., "Evolutionary art and computers," Academic Press, Harcourt, Brace, Jovanovich (1992).

[16] Ura, S., "An analysis of experiments of paired comparisons," Quality Control, Vol.16 pp.78-80 (1959) (in Japanese).

[17] Watanabe, T. and Takagi, H., "Recovering system of the distorted speech using interactive genetic algorithms," IEEE Int'l Conf. on Systems, Man and Cybernetics (SMC'95), Vol.1, pp.684-689, Vancouver, Canada (Oct., 1995) 